\title{
Best Paper Award
}

The following paper was selected by the Awards Committee of the International Symposium on Superalloys as winner of the Best Paper Award for the Tenth Symposium. The selection was based on the following criteria: originality, technical content, pertinence to the superalloy and gas turbine industries and clarity and style.

\section{High Cycle Fatigue in a Single Crystal Superalloy:}

Time Dependence at Elevated Temperature

P.K. Wright, M. Jain, and D. Cameron 


\title{
HIGH CYCLE FATIGUE IN A SINGLE CRYSTAL SUPERALLOY: TIME DEPENDENCE AT ELEVATED TEMPERATURE
}

\author{
P.K. Wright ${ }^{1}$, M. Jain ${ }^{1}$, D. Cameron ${ }^{2}$ \\ ${ }^{1}$ GE Aircraft Engines, 1 Neumann Way, Cincinnati, OH 45215, USA \\ ${ }^{2}$ Honeywell Engines Systems and Services, 111 S 34th Street, Phoenix, AZ 85072-2181
}

Keywords: Single crystal superalloys, High cycle fatigue, Environmental coatings, Rupture

\begin{abstract}
The airfoil sections of turbine blades in aircraft engines are subjected to very high temperatures, high stresses, and aggressive environments. These factors can lead to fatigue behavior that is quite complex, and dependent on stress level (both alternating and mean) and creep and environmental effects. This paper examines the high cycle fatigue (HCF) behavior of a single crystal superalloy, PWA1484 ${ }^{\mathrm{TM}}$ coated with a platinum aluminide environmental coating, at elevated temperature, $1038^{\circ} \mathrm{C}\left(1900^{\circ} \mathrm{F}\right)$, and identifies the time dependent creep and oxidation interactions of this alloy under high frequency fatigue cycling. Two major modes of failure were observed, fatigue at low mean stress, and stress rupture at higher mean stress. The capability of these basic modes were modeled separately and then combined into a damage summation model that was used to successfully describe the HCF capability under more complex cyclic conditions more representative of turbine engine conditions.
\end{abstract}

\section{Introduction}

Turbine airfoils (the portions of turbine blades in contact with the hot gas stream) are complex structures subjected to a combination of mechanical loads from centrifugal forces and gas pressures, and thermal loads from temperature gradients and transients. This paper addresses the high cycle fatigue (HCF) capability of these airfoils at a temperature representative of the hotter regions of the airfoil, $1038^{\circ} \mathrm{C}\left(1900^{\circ} \mathrm{F}\right)$.

Vibratory or HCF loads in a turbine airfoil arise from a wide range of stimuli, e.g., by the airfoil passing rapidly through alternating high and low flow regions aft of vane or nozzle segments, or by turbulent flow around the airfoil itself. They can range in frequency from a few $\mathrm{Hz}$ up to many thousands of $\mathrm{Hz}$. If the excitation frequencies are close to the one of the natural frequencies of the blade, or if the amplitude of the stimulus is high enough, then HCF failure of the airfoil can occur.

In addition to the vibratory stresses, steady-state mean stresses are also important in HCF. Cooler air from the compressor is often used for cooling turbine blades; as a result an air cooled blade develops a non-uniform temperature field which leads to steadystate thermal stresses. These are combined with mechanical stresses from centrifugal loads to give the mean stress on a blade. The vibrational loads described above are superimposed on the combination of these steady state mechanical and thermal stresses to create the total HCF environment.

In turbines, because the airfoil is very hot, the material will creep or relax with time causing the mean stresses to change over the life of the airfoil. As an example, in Figure 1, a region of an airfoil might start out at a high mean stress level, one above the level at which HCF failure would be expected (shaded area), but might relax down in stress (Path A) so that eventually it reaches a condition where HCF failure would not be expected (white area). Or the reverse might happen (Path B), with increasing mean stress over time. Finally, the vibrational amplitude as well as the mean level can be changing over time (Path $\mathrm{C}$ ), due to changing power settings in the engine.

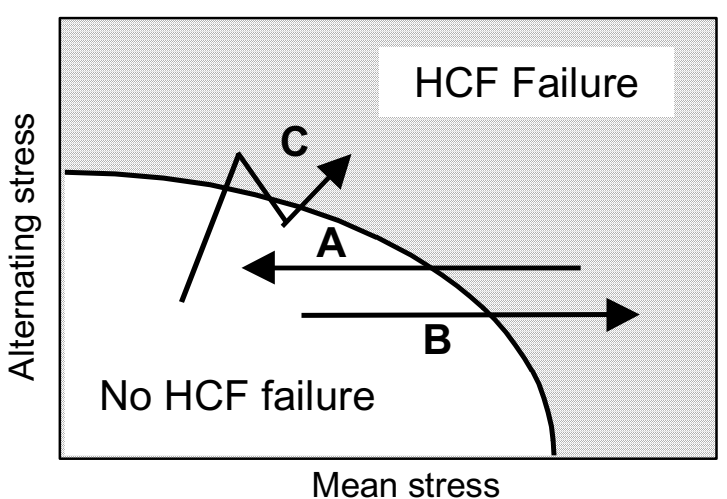

Figure 1. Illustrative HCF load paths for turbine blades

The problem to be addressed is then: How do we determine which load histories are benign and which will lead to HCF failure? To do this we need to know how damage accumulates under such variable load histories. The objective of this paper is thus to present understanding and modeling that will enable design of HCF-resistant turbine airfoils in the face of high temperature factors such as creep, a range of vibrational frequencies, and environment.

The paper will be organized as follows:

1. Experimental approach;

2. Baseline $\mathrm{HCF}$, frequency effects, and failure modes at $1038^{\circ} \mathrm{C}$ $\left(1900^{\circ} \mathrm{F}\right)$;

3. Models for $1038^{\circ} \mathrm{C}\left(1900^{\circ} \mathrm{F}\right)$ behavior;

4. Complex tests and model evaluations;

5. Discussion of results;

6. Summary \& conclusions.

This paper is based on work performed under US Air Force Research Laboratory Contract F49620-99-C-0007 to University of Dayton Research Institute, with GE Aircraft Engines, Honeywell, 
Pratt \& Whitney, and other team members as subcontractors. Dr. Ted Nicolas and Dr. Ryan Morrissey were technical program advisors, and Dr. Joseph Gallagher was UDRI program manager. Their support is gratefully acknowledged.

\section{Approach}

A combination of experimentation and analysis was performed. The experiments were all performed on a single crystal alloy, PWA $1484^{\mathrm{TM}}[1]$. This alloy was selected because one common alloy was needed for all subcontracting members of the industry team, and PWA $1484^{\mathrm{TM}}$ had been the subject of prior Air Force research [2]. All castings were made from one heat by Precision Castparts Corporation either as $1.5 \mathrm{~cm}$ ( 0.6 in) diameter rods or $1.5 \mathrm{~cm}(0.6 \mathrm{in})$ thick by $7.6 \mathrm{~cm}(3 \mathrm{in})$ wide rectangular slabs. These castings were fully heat treated using a proprietary cycle, giving the microstructure shown in Figure 2.

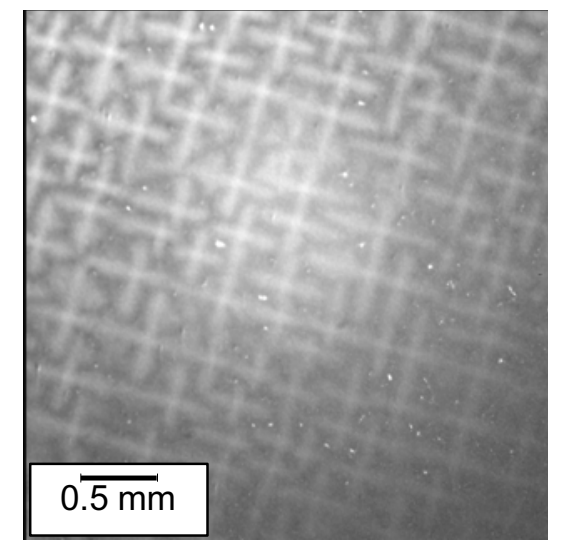

Figure 2. Microstructure of PWA1484 ${ }^{\mathrm{TM}}$ material viewed perpendicular to the casting axis showing primary dendrite spacing.

Relatively conventional specimens with a uniform cylinder gage section were machined from the castings with the specimen axis within $6^{\circ}$ of the $<001>$ crystallographic axis. Most specimens had gage sections with $0.51 \mathrm{~cm}$ (0.20 in.) diameter and $1.9 \mathrm{~cm}(0.75$ in.) length. Specimens for $900 \mathrm{~Hz}$ testing were smaller: $0.406 \mathrm{~cm}$ (0.16 in) diameter by $0.81 \mathrm{~cm}(0.32 \mathrm{in})$ gage length.

An environmentally protective platinum aluminide coating, MDC150L, was applied to all individual specimens by Howmet Thermatech Division. This produced the typical two-layer diffusion coating seen in Figure 3.

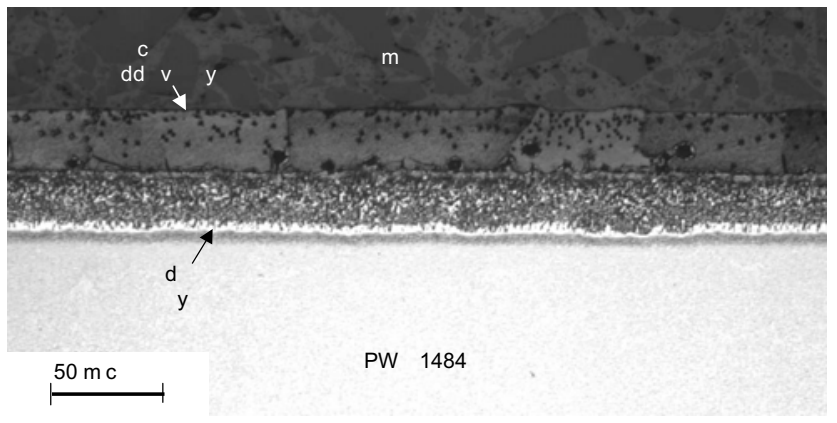

Figure 3. MDC150L PtAl coating on PWA1484 ${ }^{\mathrm{TM}}$.
This coating is typical of that used by the industry today and provides an important protective function against oxidation. Early experiments on uncoated specimens indicated a significant benefit of the coating for longer duration tests.

Most of the tests were performed at Metcut Research in Cincinnati, using closed loop hydraulic test frames and resistance furnaces for heating. GE-Aircraft Engines performed additional tests at higher frequency (up to $900 \mathrm{~Hz}$ ) on a high frequency MTS machine. Test variables included: Stress amplitude, $\mathrm{R}$-ratio $(\mathrm{R}=$ minimum/maximum stress) (from -1 to +1 ), frequency (from 6 to $900 \mathrm{hz}$ ), and a range of mean and amplitude histories that will be described later. All tests were performed at $1038^{\circ} \mathrm{C}\left(1900^{\circ} \mathrm{F}\right)$ and with both alternating and mean stress applied in an axial direction.

\section{Results}

Baseline HCF Behavior

The results for a set of baseline HCF tests performed at one frequency $(59 \mathrm{~Hz})$ and several different R-ratios will be described first. The R-ratios examined were: $-1.00,-0.33,0.10,0.50,0.80$, and 1.00 . The tests at $\mathrm{R}=1.00$ had no alternating component, thus they were actually creep-rupture tests. However, they were performed on the same equipment and specimens used for the HCF testing to eliminate potential test differences. Approximately 7 tests per R-ratio were performed. The results, plotted as test life in cycles to failure vs. stress amplitude, are shown in Figure 4. Several points are worth noting: 1) No distinct endurance limit was detected over the lifetimes examined; the S-N curves appear to be linear on a $\log$-log basis out to at least $10^{7}$ cycles. 2) With increasing R-ratio the alternating stress capability decreases, as is typical of other materials. Note, however, that the slope of the S-N curve gradually steepens as R increases. 3) The data are well-behaved with scatter typical of other directionally solidified superalloys.

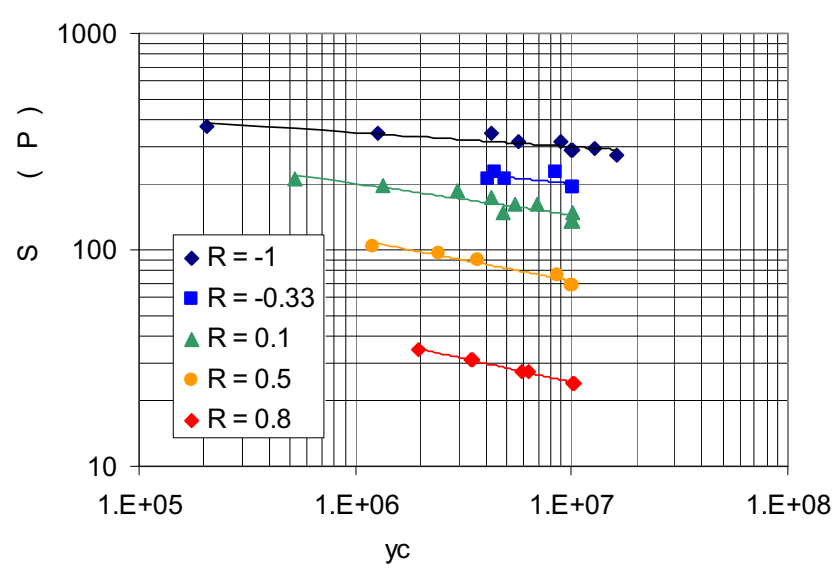

Figure 4. Cycles to fail of coated PWA $1484^{\mathrm{TM}}$ at $1038^{\circ} \mathrm{C}$ as a function of alternating stress for $5 \mathrm{R}$-ratios at $59 \mathrm{~Hz}$.

The data for $\mathrm{R}=1.0$ (rupture) are included in Figure 5 using mean stress instead of alternating stress and time to failure instead of cycles to failure. Viewed in this fashion the data at higher Rratios $(0.5,0.8$ and 1.0$)$ collapse along one line defined by the 
rupture capability $(\mathrm{R}=1.0)$, while the data at lower $\mathrm{R}$-ratio $(\mathrm{R}=$ 0.1 and below) fall well below this grouping.

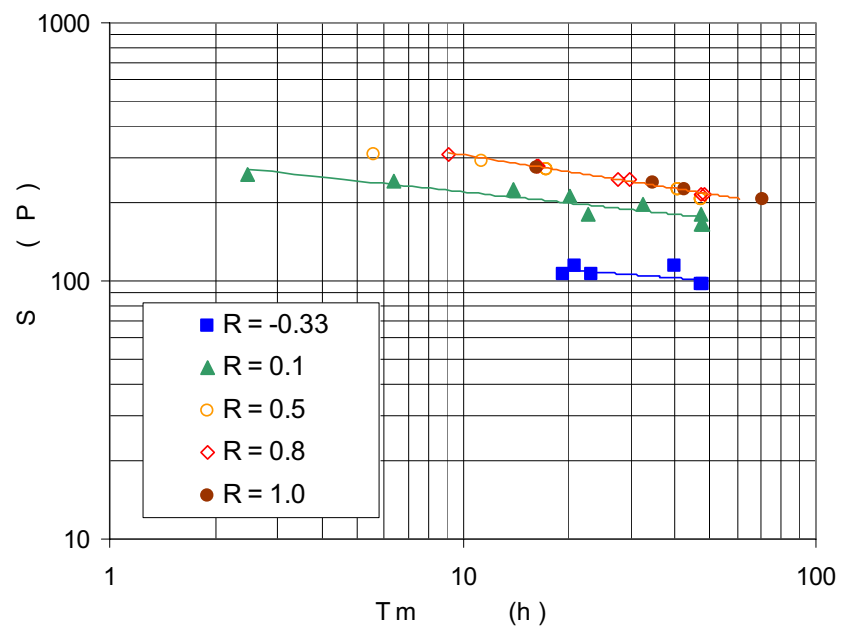

Figure 5. HCF capability of PWA $1484^{\mathrm{TM}}$ at $1038^{\circ} \mathrm{C}$ as a function of R-ratio plotted as mean stress vs. time to failure

Figure 6 shows the data from Figure 4 re-plotted as a Goodman Diagram, giving the mean and alternating stress capability for $10^{7}$ cycles life. The shape of the diagram is fairly conventional, with a gradual reduction in alternating stress capability as mean stress increases at lower mean stresses, and then a rapid decrease beyond $\mathrm{R}=0.1$ at high mean stress. The $\mathrm{R}=1$ asymptote was determined by the stress rupture capability at $47.1 \mathrm{hr}$, the time for $10^{7}$ cycles at $59 \mathrm{~Hz}$.

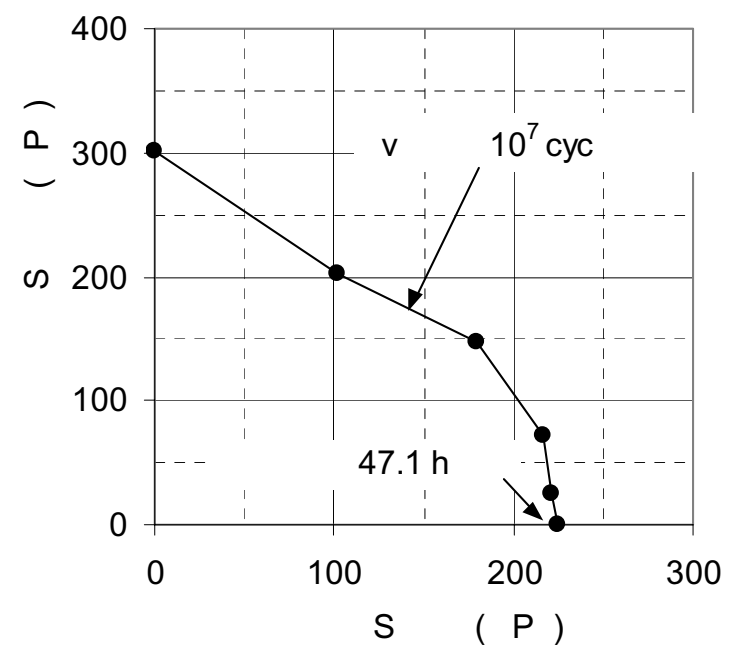

Figure 6. PWA $1484^{\mathrm{TM}}$ Goodman Diagram at $1038^{\circ} \mathrm{C}, 59 \mathrm{~Hz}$.

\section{Frequency Effects}

Tests were performed at a variety of frequencies at $\mathrm{R}=-1$ and at $\mathrm{R}=0.1$. Figure 7 shows that the results at $\mathrm{R}=-1$ are independent of frequency from 59 to $900 \mathrm{~Hz}$. This suggests that a time- independent fatigue process is happening at low mean stress, since tests at higher frequency are much shorter duration for the same number of cycles. Only the single $0.6 \mathrm{~Hz}$ test point falls off this trend, suggesting that frequency dependence may occur at very low frequencies.

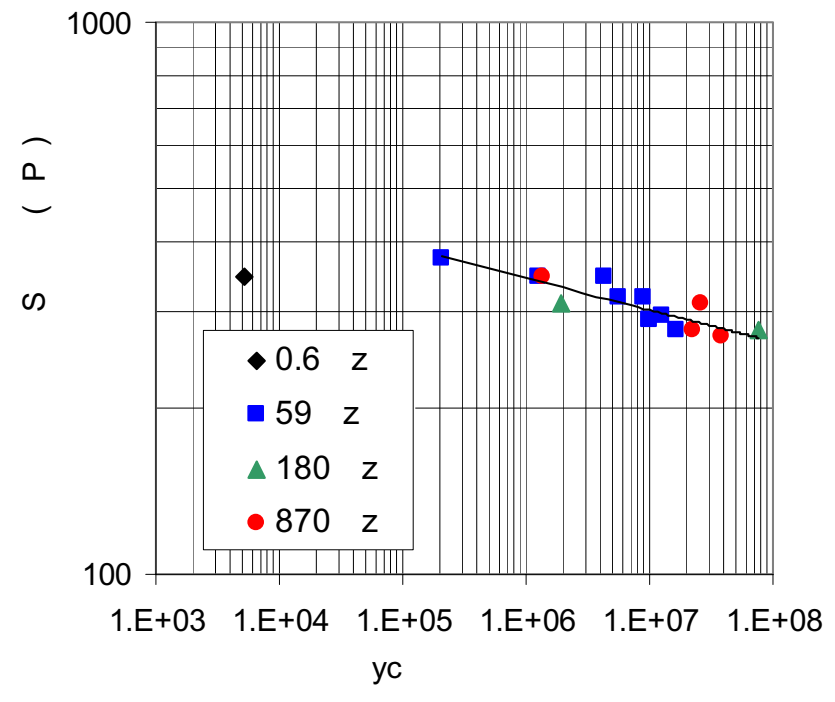

Figure 7. Alternating stress capability as a function of frequency at $\mathrm{R}=-1$.

At higher mean stress $(\mathrm{R}=0.1)$, the dependence of HCF on frequency was quite different. Figure 8 shows $\mathrm{S}-\mathrm{N}$ response at $\mathrm{R}$ $=0.1$ for frequencies of $0.6,59,250,370-400$, and $870 \mathrm{~Hz}$. At this R-ratio, there is a distinct layering of the data, with higher frequencies giving more cycles to failure at the same stress.

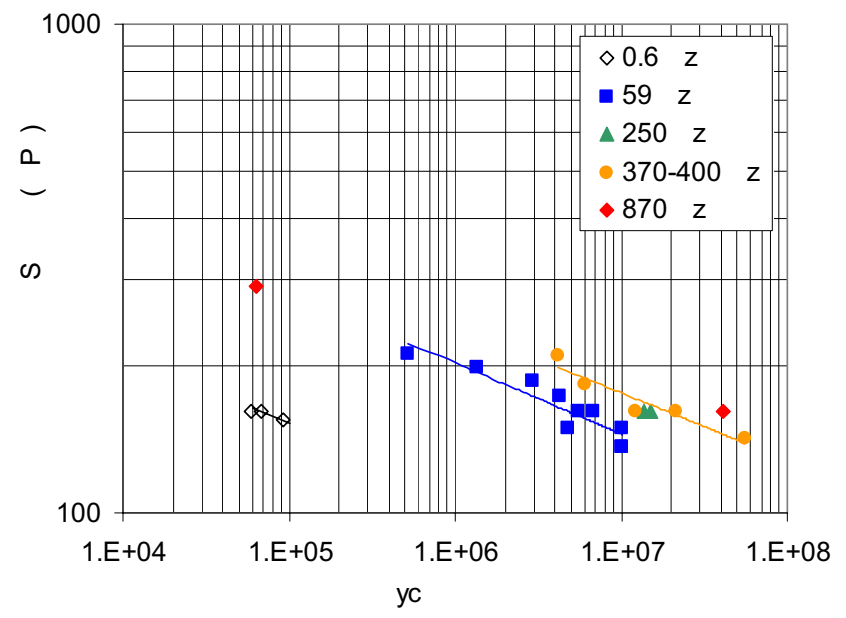

Figure 8. Alternating stress capability as a function of frequency at $\mathrm{R}=0.1$.

The results in Figure 8 are replotted in Figure 9 to show the effect of frequency on cycles to failure at a constant alternating stress level of $161 \mathrm{MPa}(23.4 \mathrm{ksi})$. As frequency increases the cycles to 
failure increase proportionately, as indicated by the $45^{\circ}$ line. This line represents a line of constant time to failure $(27.8 \mathrm{hr})$.

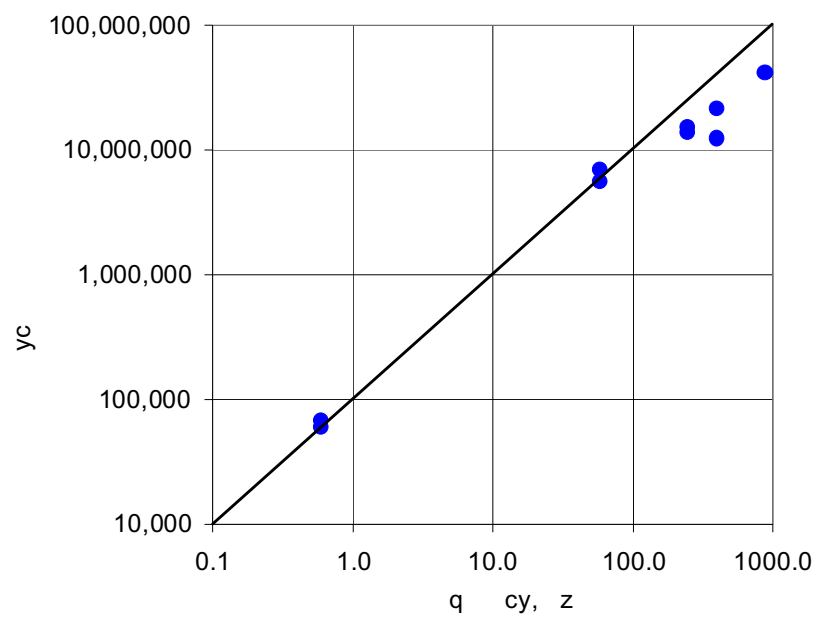

Figure 9. Failure cycles vs. frequency at $161 \mathrm{MPa}, \mathrm{R}=0.1$, $1038^{\circ} \mathrm{C}$.

That this is true can be seen by replotting the time to failure vs. frequency as shown in Figure 10. Here the fatigue capability as measured by time to failure is nearly independent of frequency. There is a slight decrease in time to failure as the frequency exceeds $100 \mathrm{~Hz}$. This suggests that the behavior appears to be transitioning toward cycle dependent behavior (sloping red line) at frequencies higher than $900 \mathrm{~Hz}$.

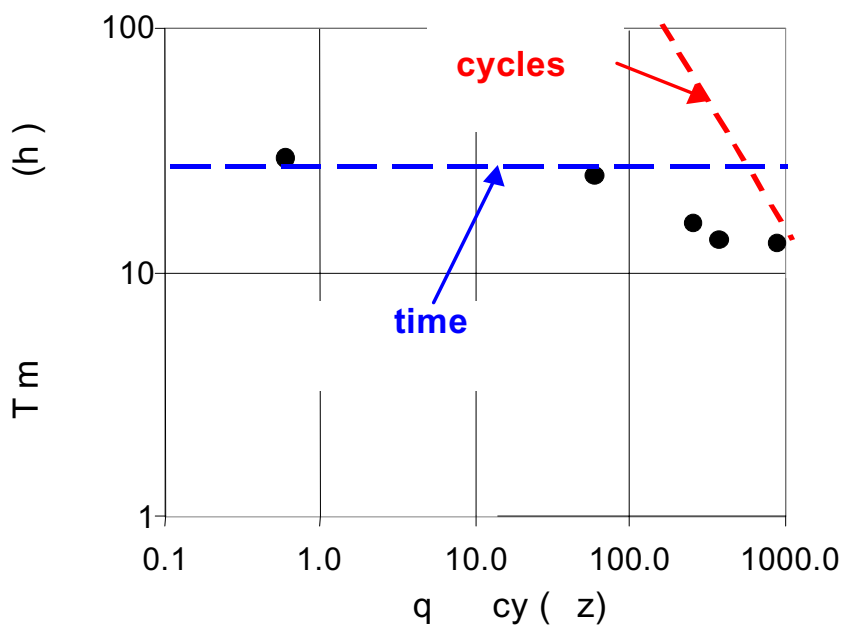

Figure 10. Failure time vs. frequency at $161 \mathrm{MPa}, \mathrm{R}=0.1$, $1038^{\circ} \mathrm{C}$

\section{HCF Failure Modes}

The appearance of these specimens after testing was illuminating. Figure 11 shows the typical modes of failure at low mean stress $(\mathrm{R}=-1)$. Figure 11a shows a specimen with an internal initiation site, typically at a carbide or eutectic gamma prime location.
Figure 11b shows a specimen in which a crack initiated from the surface, apparently from a coating crack. Both modes could sometimes be seen on a single specimen. In both cases the fracture surface in the vicinity of the origin (where most of the life was consumed) was quite flat and perpendicular to the specimen axis. Some crystallographic cracking could be seen in the fast fracture areas. In all cases there was little reduction in area or elongation of the $\mathrm{R}=-1$ specimens, and there were few if any secondary cracks seen.

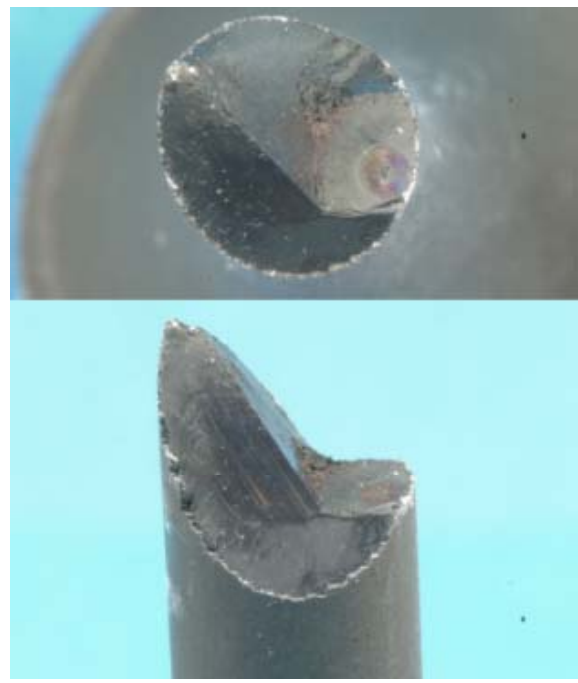

a) Internal origin

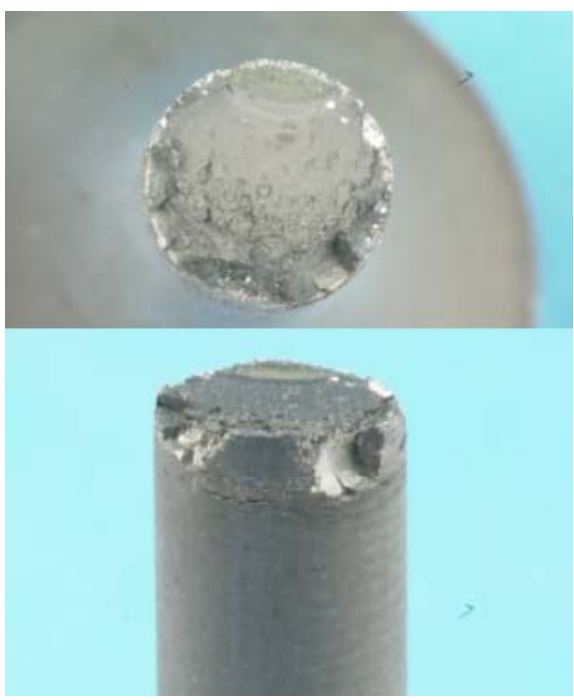

b) Surface origin

Figure 11. Fracture appearance of $\mathrm{R}=-1$ specimens at $59 \mathrm{~Hz}$.

There was a trend of tendency for internal initiation at higher stress and frequency (shorter times) and for surface initiation at lower stress and frequency (longer times) but there were exceptions to this trend. At $900 \mathrm{~Hz}$ (Figure 12b) the fracture mode transitioned to crystallographic (mixed mode) more rapidly than at lower frequency (Figure 12a). At longer failure times cracking developed in the PtAl coating, primarily at its grain boundaries, Figure 13. 


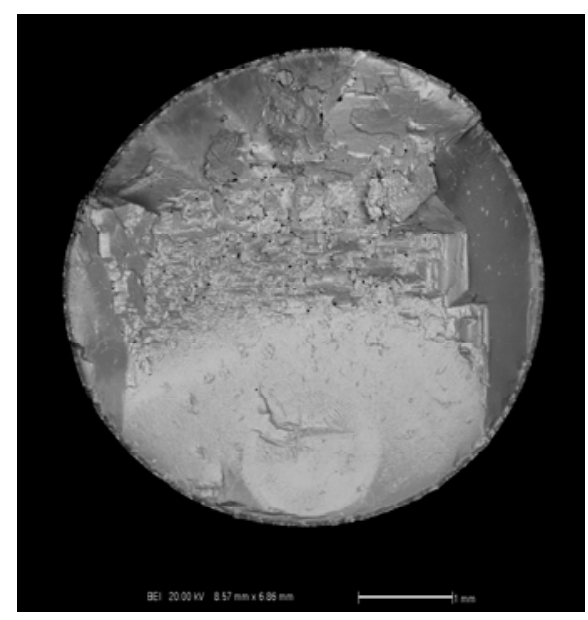

a) $400 \mathrm{~Hz}$

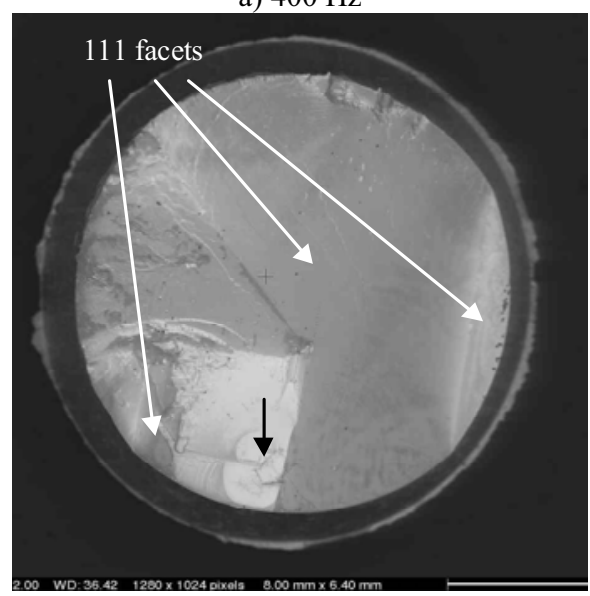

b) $900 \mathrm{~Hz}$

Figure 12. Failure appearance of $\mathrm{R}=-1$ specimens vs. frequency.

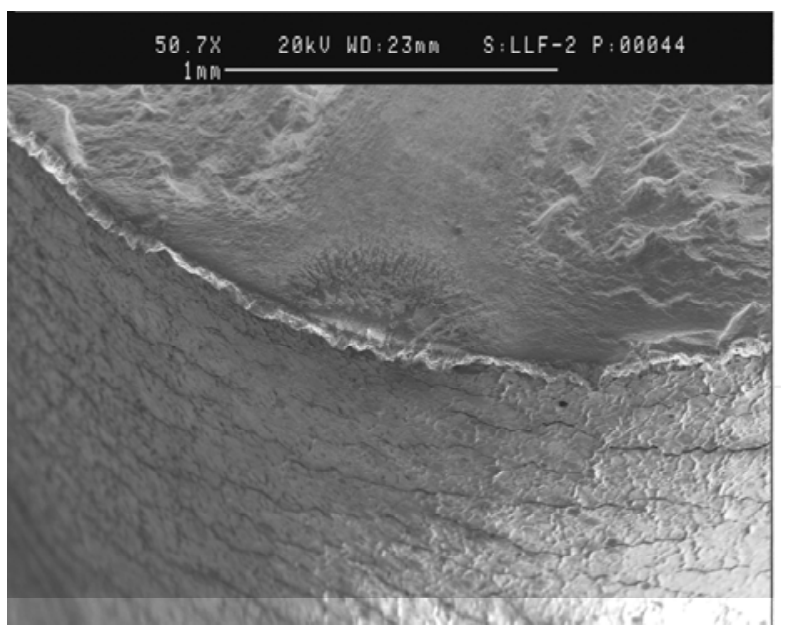

Figure 13. Coating cracking occurred in long life tests

Failure appearance of specimens tested at high $\mathrm{R}$-ratio $(\mathrm{R}=0.5$ or 0.8 ) was different, as shown in Figure 14. Some of these specimens showed significant elongation and necking, and the fracture surface was rough and origin-less (actually, many local sites of crack initiation linking up). In addition, there were many secondary surface cracks in the coating. In short, these specimens had all the characteristics of a rupture failure.

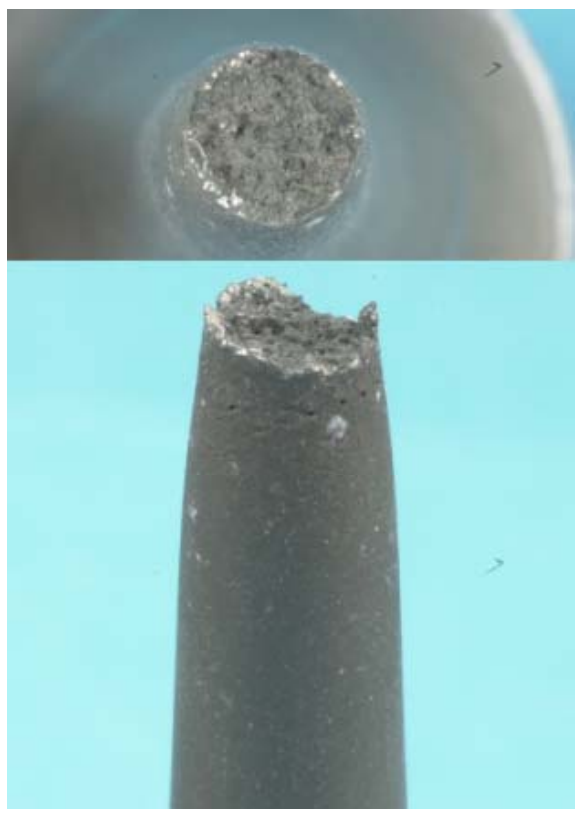

Figure 14. Fracture appearance of high R specimen.

HCF Model Development

Both the physical appearance of the specimens and their life response suggest that there are at least two major processes of $\mathrm{HCF}$ failure in this material. At low mean stress a classic timeindependent fatigue or HCF process occurs whereby a single crack initiates and then grows rapidly to failure. In contrast, at high mean stress the "HCF" failure is really one of rupture under high mean stress, with a small, perhaps negligible, role of the alternating component. At intermediate R-ratios, while the fractures are fatigue-like in appearance, the behavior is distinctly time-dependent. Here there may be some combination of the two processes acting.

As a result of these observations, we took the approach of modeling the fatigue and rupture behavior of this material separately, and then combining the two to create an overall model of mean stress and frequency-dependent behavior.

Fatigue Model: For the fatigue failure mode a power law expression for cycles to failure as a function of stress was used, Equation 1. The data shown in Figure 4 support this simple description, since they show straight lines in log-log plots.

$$
\mathrm{N}_{\mathrm{f}}=\mathrm{K}_{\mathrm{f}}\left(\sigma_{\mathrm{eq}}\right)^{\mathrm{n}}
$$

Where $\mathrm{N}_{\mathrm{f}}$ is cycles to failure and $\mathrm{K}_{\mathrm{f}}$ and $\mathrm{n}$ are experimentally fit constants. The equivalent stress, $\sigma_{\text {eq }}$, in Equation 1, is adapted from Walker [3] and accounts for the dependence of fatigue life on R-ratio as shown in Equation 2.

$$
\sigma_{\text {eq }}=\sigma_{\text {alt }}(1-R)^{(\mathrm{w}-1)}
$$


Here $\mathrm{w}$ is additional experimentally fit constant, the Walker exponent. This equivalent stress is then entered into the stress-life relationship of Equation 1 for a full description of fatigue capability. Note that the equations are independent of frequency as was shown to be the case for fatigue-driven low $\mathrm{R}$ cases.

The most significant issue with applying this model was choosing which data to use to fit the Walker exponent. Normally one chooses data over as wide a range of R-ratios as possible. However, fitting all data in this case lead to a negative value of $w$, and it also meant that the fatigue model was heavily influenced by the rupture characteristics at higher $\mathrm{R}$. Therefore all the data with $\mathrm{R}<0.1$ were used, to try to capture only those specimens failing by a fatigue mechanism. We also included the high frequency $(250-870 \mathrm{~Hz})$ results at $\mathrm{R}=0.1$. Using this approach the model constants of $\mathrm{K}_{\mathrm{f}}=1.49 \times 10^{28}, \mathrm{n}=-9.366$, and $\mathrm{w}=0.320$ were determined (stress in $\mathrm{MPa}$ ). However, as shown in Figure 9, even at $900 \mathrm{~Hz}$ at $\mathrm{R}=0.1$ a fully cycle dependent behavior has not be achieved, so using the $900 \mathrm{~Hz}$ capability may cause a slight overestimate of the R-ratio dependence of the fatigue capability.

Rupture Model: Development of a rupture damage model began with defining the stress-dependence of rupture, or $\mathrm{R}=1.0$. These data were shown previously in Figure 5. A power law expression, Equation 3, was fit to the $\mathrm{R}=1.0$ results:

$$
\mathrm{t}_{\mathrm{r}}=\mathrm{K}_{\mathrm{r}}(\bar{\sigma})^{\mathrm{m}}
$$

where $t_{r}$ is the time to failure (hr) and $K_{r}$ and $\mathrm{m}$ are experimentally fit constants. $\bar{\sigma}$ is an equivalent stress as described below. By curve fitting, $\mathrm{K}_{\mathrm{r}}=3.89 \times 10^{13}$ and $\mathrm{m}=-5.07$ (time in hr, stress in $\mathrm{MPa})$.

In the presence of a time-varying (alternating) stress component, some way is needed to relate the damage in such a cycle to that occurring when the stress is constant (rupture). Various approaches for determining $\bar{\sigma}$ for rupture were examined. The simplest approach was to assume that the alternating stress had no effect, and that only the mean stress contributed to rupture damage:

$$
\bar{\sigma}=\sigma_{\text {mean }}
$$

This will be referred to as Model A in the discussion that follows.

Other models incorporated the effect of a time-varying mean + alternating stress, Equation 5, by integrating the rupture damage over the stress cycle. This was done by dividing the cycle into small, constant stress time increments (100 per cycle) and determining the rupture damage as a fraction of the time spent at that stress compared to the rupture time to failure at that stress.

$$
\sigma(\mathrm{t})=\sigma_{\mathrm{m}}+\sigma_{\mathrm{a}} \sin (2 \pi \mathrm{t} / \tau)
$$

For the failure time, $t_{r}$, the total rupture damage, $D_{R}$, in a given cycle of period $\tau$ was set to 1 :

$$
D_{R}=1=\int_{0}^{\tau} d t / t_{r}(\sigma(t))
$$

and the time or cycles to failure $\left(\mathrm{N}_{\mathrm{f}}=\mathrm{t}_{\mathrm{r}} \mathrm{x}\right.$ f) were computed to satisfy this criterion. Using this approach, Model B treated compressive stress excursions (when $\mathrm{R}<0$ ) the same as tensile stress; that is, compressive stresses contributed to damage the same as tension. Model $\mathrm{C}$ treated compression as non-damaging; time spent in compression was ignored. These two models give the same results for $\mathrm{R}>0$ since no compression exists for these conditions. For Models $\mathrm{B}$ and $\mathrm{C}, \bar{\sigma}$ is not a simple analytic function; instead the damage integrals in Equation 6 were evaluated numerically.

The results of these various models are shown on the Goodman Diagram of Figure 15 along with the experimentally determined $10^{7}$ cycles (or $47.1 \mathrm{hr}$ ) stress capabilities at $59 \mathrm{~Hz}$. The predictions of Models $\mathrm{B}$ and $\mathrm{C}$, the rupture damage approaches using time-varying stress, are notable. The ability of these models to describe HCF capability extends over a large portion of the Goodman Diagram, even into the region where failure mode observations suggest rupture is not the dominant failure mode $(\mathrm{R}$ $<0)$. The Rupture Model A is satisfactory at high mean stress conditions, but as $\mathrm{R}$ decreases it over-predicts capability significantly. The capability of the Walker-based fatigue model is good over the range expected (up to $\mathrm{R}=0$ ) but for $\mathrm{R}$ above that it significantly over-predicts capability. This is consistent, however, with a change in failure mode from fatigue to rupture-dominated at higher R.

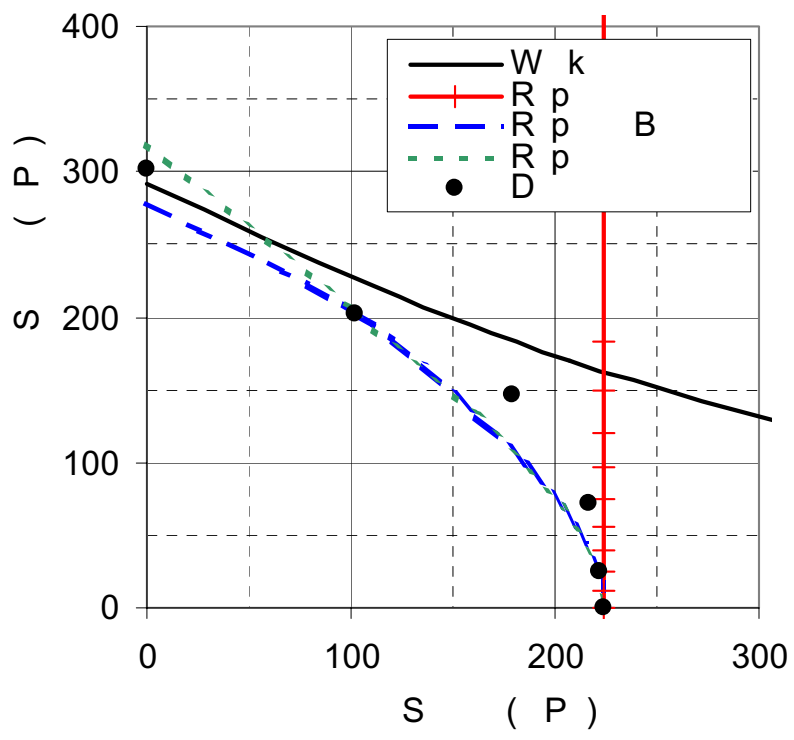

Figure 15. Model predictions of Goodman Diagrams vs data.

Combined Damage Models

To help evaluate these models, additional tests containing more complex conditions were performed. 10 tests were performed with two segments at different conditions. These included: 1) Pure rupture $(\mathrm{R}=1.0)$ to $50 \%$ of life followed by pure fatigue $(\mathrm{R}$ $=-1.0$ ) to failure; 2) Pure fatigue to $50 \%$ of life followed by pure rupture to failure; 3) Fatigue at one R-ratio to $50 \%$ of life followed by fatigue at another R-ratio to failure. An additional 8 tests were performed with a constant alternating stress amplitude and a continuously increasing or decreasing mean stress to failure (Path A or B of Figure 1). Finally, 20 constant mean and 
alternating stress test results at different frequencies described previously were included. Except for the high frequency $\mathrm{R}=-1$ data, these tests had not been used to fit the models.

The results of the 2-segment tests with rupture followed by fatigue were particularly instructive. The second legs of these tests showed fatigue lives were reduced by the prior rupture exposure. (Similar results were obtained on a larger number of uncoated specimens.) This suggests that rupture and fatigue are not independent processes, but interact. The simplest interaction model is linear damage summation, as shown in Equation 7:

$$
1=D_{R}+D_{F}=\left(N / N_{f}\right)+\left(t / t_{r}\right)
$$

This model was evaluated for the 2-segment tests with $t_{r}$ calculated by Rupture Model A and results are shown in Figure 16. If no damage interaction had occurred, the test results would have been expected to cluster around lines of $D_{F}=1$ or $D_{R}=1$. However, most of the 2-sgment tests cluster around the 45-degree line predicted by a linear damage model. The tests in which fatigue is followed by rupture appear to be an exception, but the deviation is not large.

Using the simple, one-mode models from the previous section, and the linear cumulative damage rule, Eq. 7, the capability of the models, either individually or in combination, was examined using all of the 67 test results for which failures were obtained (run-outs were omitted).

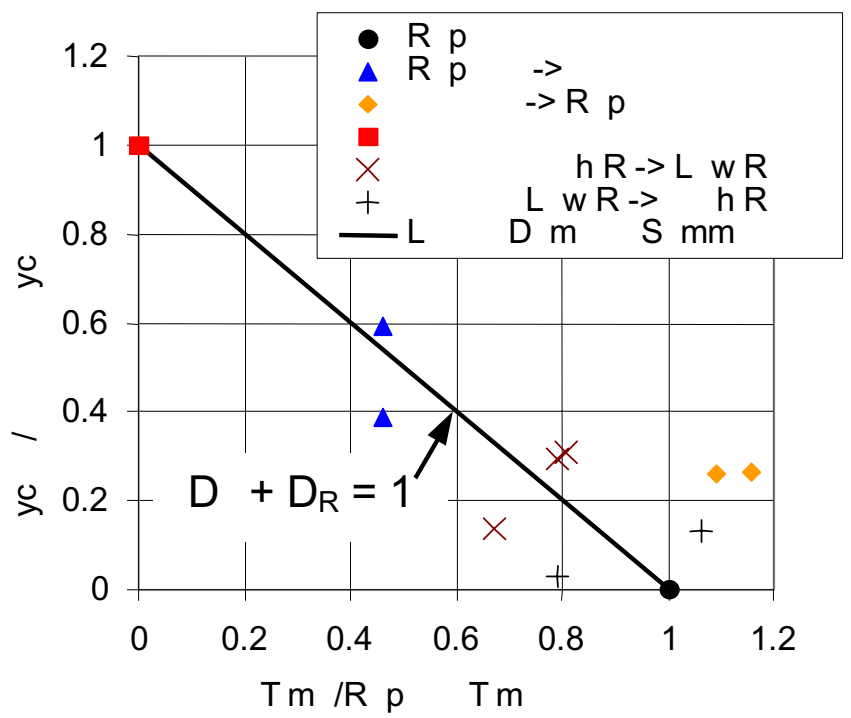

Figure 16. Damage plot of 2-segment tests using Walker Fatigue and Rupture Model A.

The ability of the models to describe the data set was evaluated in three ways:

1) Computation of the average total damage, $D_{\text {tot }}$, for all the tests included in each model. This gives a measure of the average bias of the model. Ideally $\mathrm{D}_{\text {tot }}$ should be 1.0 .

2) Computation of the standard error of model fit, $\mathrm{s}_{\mathrm{e}}$ : $s_{e}=\sqrt{\frac{\sum\left[\log \left(N_{f}^{i}(\text { pred })\right)-\log \left(N_{f}^{i}(o b s)\right)\right]^{2}}{(M-P)}}$

where $\mathrm{N}_{\mathrm{f}}^{i}$ (pred) is the predicted cycles to failure and $\mathrm{N}_{\mathrm{f}}^{i}(\mathrm{obs})$ is the observed cycles to failure of the $i$ th test, $\mathrm{M}$ is the total number of tests (67) and P is the number of constants used to fit the models ( $\mathrm{P}$ ranged from 2 to 5 ). $\log \left(\mathrm{N}_{\mathrm{f}}\right)$ was used because of the power law stress-life relationships identified. An indication of the inherent scatter in the data was obtained by determining the lumped $\mathrm{s}_{\mathrm{e}}$ of all the baseline $59 \mathrm{~Hz} \mathrm{HCF}$ tests from Figure 4 about their own average power law curve fits (one for each of the $5 \mathrm{R}$ ratios). This computation led to a $s_{e}=0.189$. This represents approximately the smallest $s_{\mathrm{e}}$ that could be expected from fitting models to this data set.

3) Visual examination was made of the clustering of the predicted failure times in a predicted vs. observed life plot such as Figure 17. This identified any bias in the model in favor of or against any particular test condition. Ideally, the average of all subgroups would be the same as the total average, and all conditions would scatter similarly around the predicted mean.

The number of combinations of models is too large to present in totality here, so a discussion of only the more promising or instructive ones will be done. Table 1 shows average $s_{e}$ and $D_{\text {tot }}$ for 7 models or combinations. The Walker HCF model alone did not fit the data well, as evidenced by its large $\mathrm{s}_{\mathrm{e}}$ and ave $\mathrm{D}_{\text {tot }}$ less than 1.0. The $s_{\mathrm{e}}$ for Rupture A could not be evaluated since this model predicted infinite lives for $\mathrm{R}=-1$ conditions. Also, its ave $\mathrm{D}_{\text {tot }}$ is quite far from 1.0.

Rupture B alone, considering only integrated rupture damage (including compression), has $\mathrm{D}_{\text {tot }}$ close to 1.0 and the smallest $\mathrm{s}_{\mathrm{e}}$ (best fit) of models examined. This is striking since many of these tests were conducted in regime where a combination of fatigue and rupture effects would be expected. Model C was not quite as good as B.

Table 1. Comparison of model predictions

\begin{tabular}{|l|c|c|}
\hline \multicolumn{1}{|c|}{ Model } & $\mathbf{s}_{\mathbf{e}}$ & ave $_{\text {tot }}$ \\
\hline Walker HCF (no rupture damage) & 1.426 & 0.679 \\
Rupture A (mean stress damage) & large & 0.333 \\
Rupture B (integrated over cycle; & 0.418 & 1.008 \\
$\quad$ compression damaging) & & \\
Rupture C (integrated over cycle; & 0.509 & 0.913 \\
$\quad$ compression not damaging) & & \\
Walker HCF + Rupture A & 0.450 & 0.999 \\
Walker HCF+ Rupture B & 0.426 & 1.556 \\
Walker HCF + Rupture C & 0.427 & 1.642 \\
\hline
\end{tabular}

However, examining the predicted vs. observed behavior of the integrated rupture damage models ( $\mathrm{B}$ and $\mathrm{C}$ ) shows that, not surprisingly, they do a poor job of describing the material capability in HCF-dominated conditions, such as $\mathrm{R}=-1$, or 900 $\mathrm{Hz} \mathrm{HCF}$. As shown in the predicted vs. observed cycles to 
failure plot of Figure 17, these conditions are significantly overpredicted (the models are non-conservative). This over-prediction is not surprising since Model B is based purely on rupture capability, while the behavior at this low mean stress and high frequency is governed by a fatigue mode (see "HCF Failure Modes"). Since low mean stress, high frequency conditions can be important in turbine blade HCF, the use of the integrated rupture models alone is not recommended.

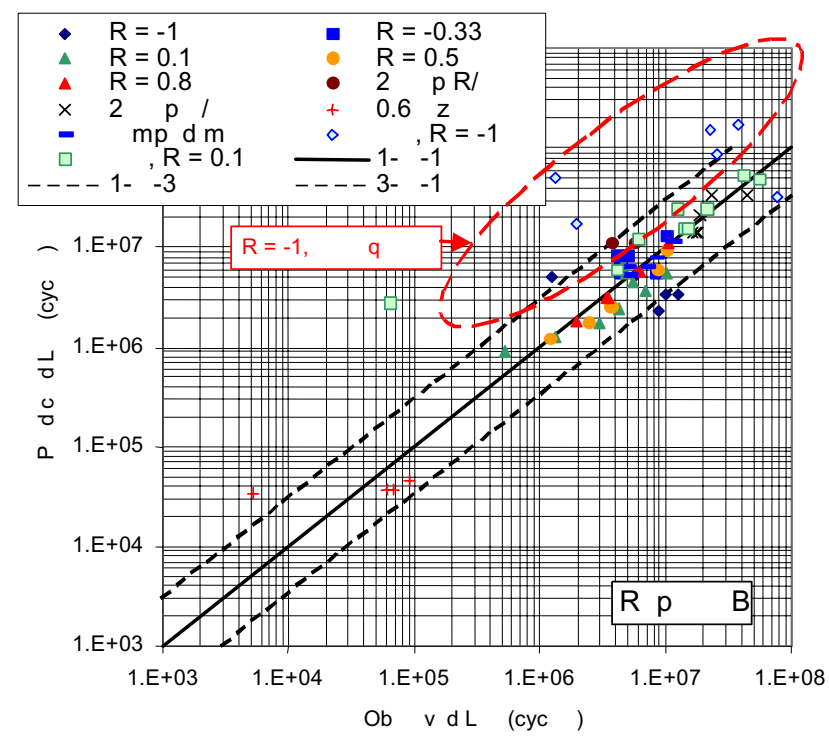

Figure 17. Predicted vs. observed lives for Rupture Model B for all tests.

A linear combination of the Walker fatigue model along with the mean stress rupture model (Model A) gives a reasonably small standard error of fit (Table 1) and a $\mathrm{D}_{\text {tot }}$ very close to 1.0. A plot of the predicted vs. observed test lives for Walker + Rupture A is shown in Figure 18.

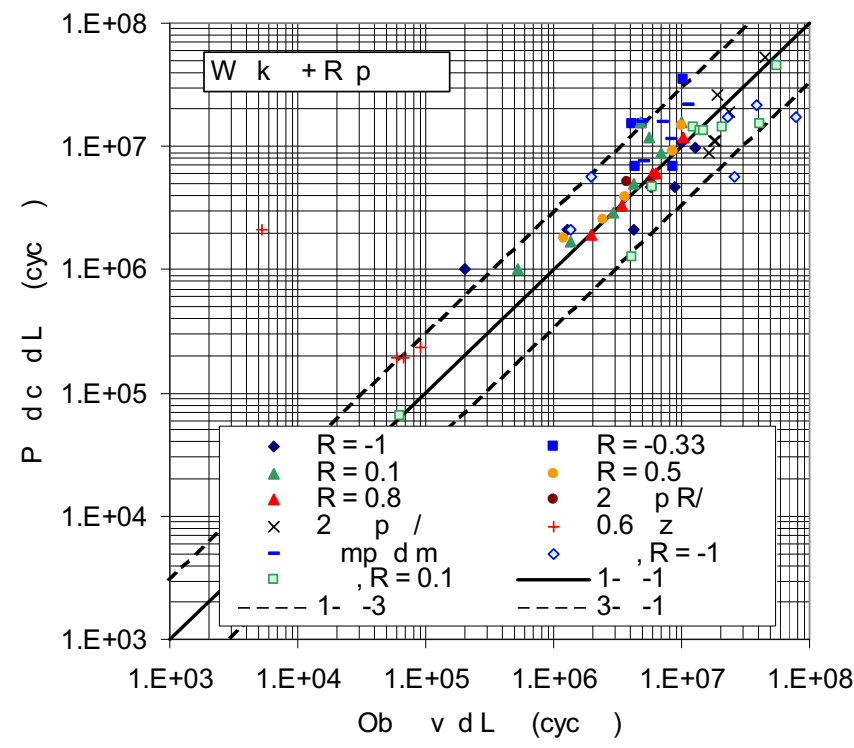

Figure 18. Predicted vs. observed lives for all tests for Walker $\mathrm{HCF}+$ Rupture A combined model.
Visually, the scatter about the ideal 1-to-1 in Figure 18 appears to be similar to that seen in Figure 17, and there is little or no bias by test condition or type. Thus, the low mean stress or high frequency HCF tests that were poorly predicted by the integrated rupture damage models are predicted much better by the combined fatigue + rupture damage A approach. This improvement occurs because a combined approach allows a frequency-independent fatigue approach in the low mean stress regime along with a frequency dependent rupture-based approach in the high mean stress regime. The increase in the proportion of rupture damage at failure with R-ratio is shown in the percentages listed above each data point Figure 19. The contribution of rupture damage is seen to be significant at $\mathrm{R}=0.1$ and above, while fatigue damage dominates below $\mathrm{R}=0.1$.

A $59 \mathrm{~Hz}$ Goodman Diagram was calculated for the Walker + Rupture A Model and is shown in Figure 19. It shows good agreement with the observed $10^{7}$ HCF capability, as expected from the general agreement of this model with the individual test points shown in Figure 18. The slight under-prediction of the $\mathrm{R}=$ -1 point and over-prediction of the $R=-0.33$ and 0.1 points in Figure 19 is due to other HCF data included in the model fitting shown in Figure 18 but not shown in Figure 19 that have influenced the shape of the model in this region.

The only test condition poorly predicted by this model is $0.6 \mathrm{~Hz} \mathrm{R}$ $=-1$, and as noted above, this condition is not of engineering significance.

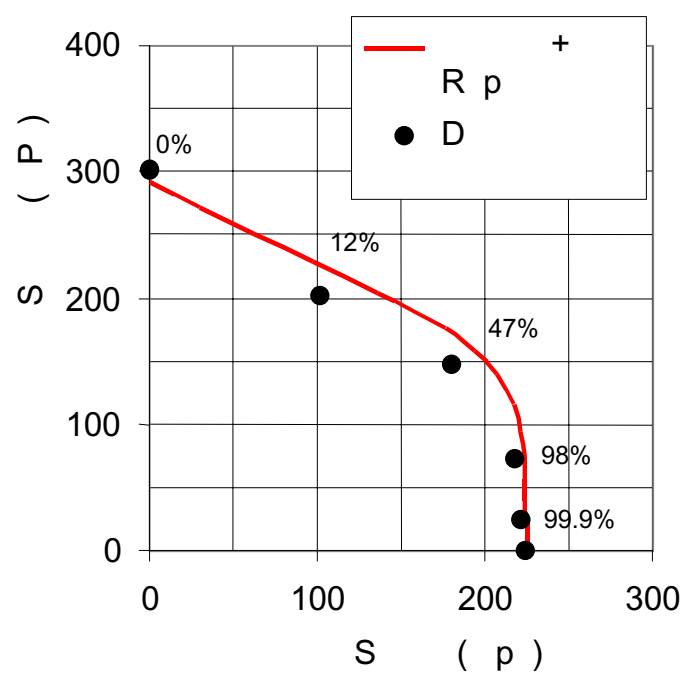

Figure 19. Goodman Diagram at $59 \mathrm{~Hz}$ predicted by Walker Fatigue + Rupture A Model.

The $\mathrm{s}_{\mathrm{e}}$ 's of the two other linearly combined models shown in Table 1 (Walker + Rupture B and Walker + Rupture C) are very close to that of Walker + Rupture A, but their average total damage, ave $\mathrm{D}_{\text {tot }}$, is too high, and they are somewhat more complicated to implement. 


\section{Discussion}

Mechanisms of time dependence

The time or frequency dependence observed here can arise from either creep-rupture or environmental effects (or both). Rupture clearly contributes to time dependence in this material since the frequency dependence is R-ratio (mean stress) dependent where no change in environment has occurred. Differences in results between tests on coated and uncoated specimens (not reported here) suggest that there is also some environmental dependence, however. Certainly oxidation was observed on the fracture surfaces of even the coated specimens. The difference in life between coated and uncoated material is minimal for short lives but increases as lives increase (stress decreases). However, the HCF-rupture cumulative damage models seem to capture much of the time dependence of HCF behavior without explicitly considering environmental effects. This may be because the primary contribution of environment in coated material occurs in the crack growth portion of life (in the less oxidation resistant superalloy substrate) and typically in HCF crack growth occupies a smaller fraction of total life than does crack initiation (coating penetration).

\section{$\underline{\text { Rupture-fatigue interactions }}$}

The test results and modeling strongly suggest that some sort of interaction between rupture and fatigue occurs for single crystal superalloys at high temperatures. The mechanism of this interaction was not evident from SEM examination of the fracture surfaces or polished sections after testing. In the region where rupture and fatigue appear to be most strongly interacting $(\mathrm{R} \approx 0)$ the fracture appearance is much more reminiscent of fatigue than rupture. It may be that the interaction effect occurs primarily during the longer crack initiation stage and that the shorter propagation stage is dominated by fatigue.

\section{Transition frequency}

A transition between time dependent and time independent fatigue behavior was observed at two R-ratios, -1 and 0.1 , as illustrated in Figures 7 and 10 . The transition frequency appeared to be strongly dependent on mean stress, since at $\mathrm{R}=-1$ it was below $59 \mathrm{~Hz}$, while at $\mathrm{R}=0.1$ it was above $870 \mathrm{~Hz}$. The shift in transition frequency is consistent with the modeling approach adopted, since with increased rupture contributions at higher mean stress, the cyclic frequency must be raised to achieve fatigue dominated failure. The modeling approach does not predict a transition frequency at $\mathrm{R}=-1$, since all frequencies at that R-ratio are assumed to result in pure fatigue. The limited data at $0.6 \mathrm{~Hz}$, $\mathrm{R}=-1$ suggest there may be a low frequency transition of unknown origin. Fortunately, such low frequency conditions are usually not of interest to turbine blade $\mathrm{HCF}$, so as a practical matter this discrepancy may have little significance.

\section{Endurance limit behavior}

While is it customary to treat $\mathrm{HCF}$ as a property which possesses an endurance limit, that is, an alternating stress level below which failure will never occur, the results obtained here do not support that concept at these conditions. If a true endurance limit exists at $1038^{\circ} \mathrm{C}$, it is well beyond $10^{8}$ cycles. Samples removed from test without failure after approximately $10^{7}$ cycles often showed evidence of damage in the form of surface cracks, as illustrated in Figure 20.

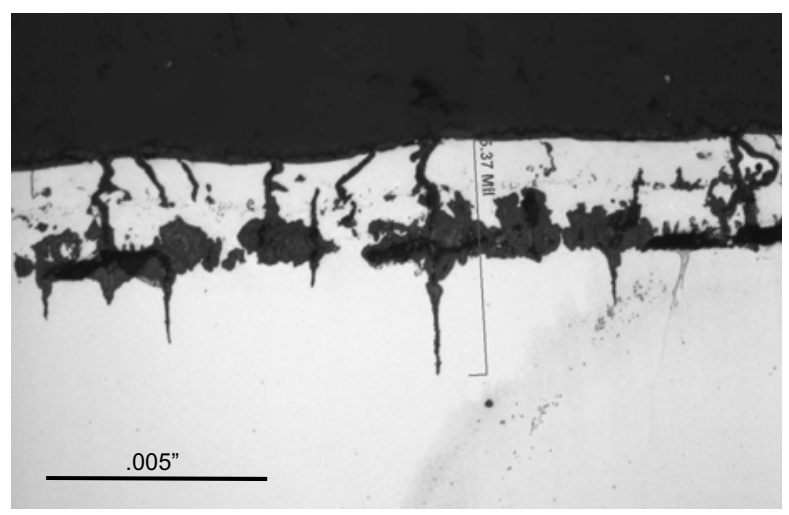

Figure 20. Surface cracking observed in run out specimen after $16.3 \times 10^{6}$ cycles at $\mathrm{R}=-1,59 \mathrm{~Hz}, 276 \mathrm{MPa}$ alt. stress.

Here the cracks have penetrated the coating and are growing into the substrate alloy, suggesting that further cycling will cause further growth and eventual failure.

\section{Identification of fatigue model constants}

The most difficult part of evaluating the above models was identifying the fatigue model constants. The modeling results were quite sensitive to the fatigue model constants used. To achieve a good fit of all the results, the test data used to fit the fatigue model must be totally or mostly free of rupture influences. Going in to this project we did not anticipate the large extent of rupture influence at $1038^{\circ} \mathrm{C}$ and thus had barely adequate data to satisfactorily identify the pure fatigue capability. Having sufficient data at multiple R-ratios at low mean stress and as high a frequency as possible is key to being able to identify a good fatigue model.

HCF behavior at other temperatures and frequencies

While testing in this study was performed at only one temperature, it can be inferred from the principles behind the models that similar effects would be expected over a wide range of elevated temperature, down to at least $850^{\circ} \mathrm{C}$ and possibly as low as $760^{\circ} \mathrm{C}$. Observations of $\mathrm{HCF}$ in other alloys support this. Typically the rupture mode of failure is more temperature dependent than the fatigue mode, so at lower temperatures the Goodman diagram becomes more elongated (the mean stress axis intercept moves to the right) and at higher temperatures it becomes more truncated (more rounded). This characteristic is shown in Figure 21 for $\mathrm{HCF}$ data for single crystal superalloy Rene N5 (4) at $870^{\circ} \mathrm{C}$ and $1093^{\circ} \mathrm{C}$. The $10^{7}$ cycle stress levels on both axes have been normalized by the $871^{\circ} \mathrm{C} \mathrm{R}=1$ capability to show the relative shapes of the curves and the magnitude of change between $871^{\circ} \mathrm{C}$ and $1093^{\circ} \mathrm{C}$. The model curves have been constructed using the Walker + Rupture Model A approach as described above. Except for the data point at $\mathrm{R}=0.6,871 \mathrm{C}$, the agreement between the model and the data is good; not surprising since the location and shape of the curves depend on data at $\mathrm{R}=-$ 1,0 , and 1 . Prediction of the $\mathrm{R}=0.6$ point at $871 \mathrm{C}$ requires a long extrapolation of the mean stress dependence of fatigue, which probably explains why the agreement is not good. Figure 21 shows that, as expected, increasing temperature decreases the mean stress (rupture) capability faster than the alternating stress (fatigue) capability, and causes rupture to influence a larger fraction of the Goodman Diagram. 
While fitting these data by this method seems somewhat simplistic, there is considerable power behind it since the model can be used for other cyclic frequencies and for complex loading histories, as demonstrated in Figure 18. In an analogous argument, the transition frequency at any fixed R-ratio is also expected to depend on temperature.

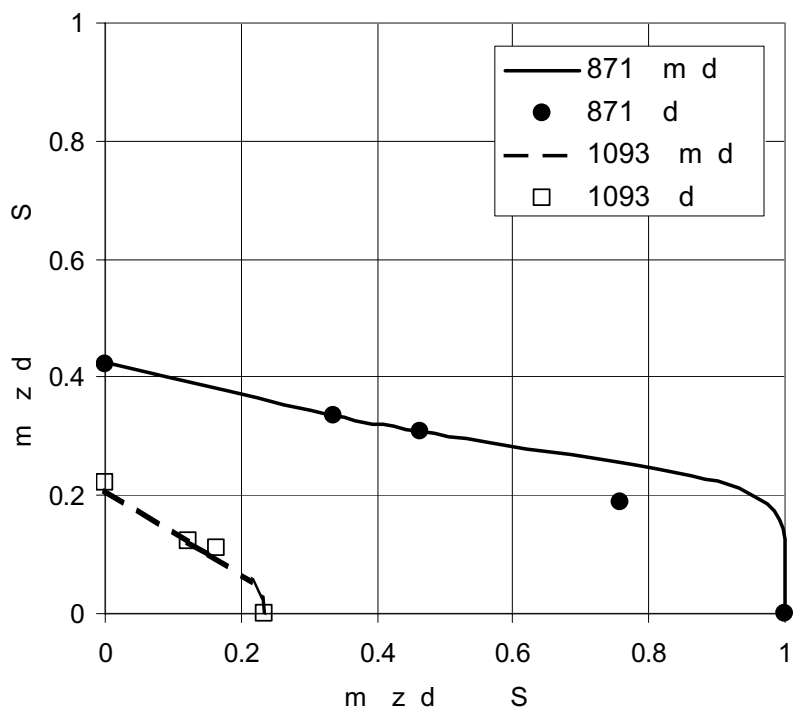

Figure 21. HCF Goodman Diagrams of Rene N5 at $871^{\circ} \mathrm{C}$ and $1093^{\circ} \mathrm{C}$ normalized by the $871^{\circ} \mathrm{C} \mathrm{R}=1$ capability.

\section{Summary and Conclusions}

Two primary modes of failure control the HCF capability at $1038^{\circ} \mathrm{C}$ (and other elevated temperatures). At low mean stress ( $\mathrm{R}$ less than $\sim 0$ ) and high frequency, a fatigue process is dominant. Fatigue-like crack initiation and flat crack growth (from internal or surface sites) are seen. At higher mean stress or lower frequency the failure process is dominated by creep rupture where failure proceeded or aided by rupture-like multiple crack nucleation. The influence of the rupture mode can occur over a wide portion of the Goodman Diagram; at $1038^{\circ} \mathrm{C}$, damage at $\mathrm{R}=$ 0.1 is approximately $50 \%$ rupture.

Frequency effects are different in the two failure modes. In the fatigue regime frequency has little or no effect on HCF capability. In the rupture mode, cyclic life increased as frequency increased; time to failure was constant. There exists a transition frequency (range) from fatigue to rupture that is dependent on $\mathrm{R}$ (and temperature). The time dependence of $\mathrm{HCF}$ at $1038^{\circ} \mathrm{C}$ appears to result from a combination of rupture and environmental effects although rupture appears to have the strongest influence.

Rupture and HCF interact, as shown by 2-leg tests and by the ability of the combined Rupture + Fatigue models to describe the life capability. Modeling fatigue and rupture damage separately (using Walker and mean stress Rupture A expressions, respectively), and then summing the damage contributions using a Linear Damage Rule describes HCF life capability of a wide range of tests well even for complex load histories. This approach appears to be simple and accurate enough to be suitable for use in turbine blade airfoils.

\section{References}

1. A.D. Cetel and D.N. Duhl, Superalloys 1988, Proc. of the $6^{\text {th }}$ International Symposium on Superalloys, Seven Springs, TMS (1988), p235ff.

2. S. E. Cunningham, D.P. DeLuca, and F.K. Haake, ${ }_{2}$ rack Growth and Life Prediction in Single Crystal Nickel Superalloys, Volume 1, WL-TR-94-4089, Feb 1996, Wright Patterson AFB.

3. K. Walker, "The Effect of Stress Ratio During Crack Propagation and Fatigue for 2024-T3 and 7075-T6 Aluminum", Effects of Environment and Complex Load History on Fatigue Life, ASTM STP621, 1970, pp1-14.

4. C.S. Wukusick, L. Buchakjian, "Property Balanced Nickel Base Superalloys for Producing Single Crystal Articles", U.S. Patent 6,074,602, (2000). 